\title{
Snail Is an Independent Prognostic Indicator for Predicting Recurrence and Progression in Non-Muscle Invasive Bladder Cancer
}

\author{
Yuancheng Gou ${ }^{\mathrm{a}, \mathrm{d}}$, Weihong Ding ${ }^{\mathrm{a}, \mathrm{d}}, \mathrm{Ke} \mathrm{Xu}^{\mathrm{a}}$, Hong Wang ${ }^{\mathrm{b}}$, Zhongqing Chen ${ }^{\mathrm{b}}$, \\ Jun Tanc, Guowei Xia ${ }^{\mathrm{a}, \mathrm{e}}$, Qiang Ding ${ }^{\mathrm{a}}$
}

\begin{abstract}
Background: Snail, an inducer of the epithelial-to-mesenchymal transition (EMT) increases motility and invasiveness of cancer cells by repressing E-cadherin expression. We investigate the relationship between Snail expression and clinicopathological parameters, and evaluate its prognostic significance in patients with non-muscle invasive bladder cancer (NMIBC).
\end{abstract}

Methods: Three hundred thirty-two patients treated with transurethral resection of the bladder tumor (TURBT) between October 2002 and July 2010 were histopathologically confirmed to be NMIBC. Tumor recurrence and progression were followed up in all patients. Immunohistochemical staining of 332 slices was performed. The expression of Snail was evaluated by IHC and graded for intensity and area of staining. We correlated Snail scores with clinical and pathological variables, and association of Snail staining with tumor recurrence and progression was evaluated by univariate, multivariate analysis and Kaplan-Meier survival curves.

Results: Of 332 patients with NMIBC, there was Snail positivity in 104 tumors $(31.3 \%)$, and Snail expression correlated with age, multifocality, carcinoma in situ (CIS), tumor stage and tumor grade (each $\mathrm{P}<0.05$, respectively). A multivariate Cox regression model revealed that Snail expression was an independent predictor of tumor recurrence (hazard ratio $(\mathrm{HR}): 1.95, \mathrm{P}=0.001$ ) and progression (HR: 2.34, P = 0.014) in patients with NMIBC. Kaplan-Meier estimates showed Snail expression was significantly associated with

Manuscript accepted for publication July 31, 2014

\footnotetext{
a Department of Urology, Huashan Hospital affiliated to Fudan University, Shanghai 200040, China

${ }^{b}$ Department of Pathology, Huashan Hospital affiliated to Fudan University, Shanghai 200040, China

${ }^{\mathrm{c} D e p a r t m e n t}$ of Biostatistics \& Social Medicine, School of Public Health, Fudan University, Shanghai 200032, China

${ }^{\mathrm{d}}$ These authors contributed equally to this study.

${ }^{\mathrm{e}}$ Corresponding Author: Guowei Xia, Department of Urology, Huashan Hospital affiliated to Fudan University, No. 12 Middle Urumqi Road,

Shanghai 200040,China. Email: guowei_xia@126.com
}

recurrence and progression (log-rank test, $\mathrm{P}<0.0001$, respectively).

Conclusions: Analysis of Snail expression in 332 NMIBC tissue specimens revealed its potential usefulness as a biomarker to predict the NMIBC prognosis.

Keywords: Snail; EMT; NMIBC; Prognosis; Immunohistochemistry

\section{Introduction}

Snail is a member of a super family of zinc-finger transcription factors, which was first identified in Drosophila melanogaster [1]. It has two consensus phosphorylation sites phosphorylated by GSK-3beta to dually regulate the function of this protein. Phosphorylation of the first motif regulates its beta-TrCP-mediated ubiquitination, whereas phosphorylation of the second motif controls its subcellular localization [2-4]. It has been found to negatively correlate with E-cadherin expression in various epithelial cancers triggering a complete epithelial-to-mesenchymal transition (EMT) and thereby of tumor progression and metastasis [5-7]. Bladder cancer is estimated to be the ninth most common cause of cancer worldwide and the 13th most numerous cause of death from cancer $[8,9]$. Approximately $75-85 \%$ of patients with bladder cancer present with disease confined to the mucosa (stage Ta, carcinoma in situ (CIS)) or submucosa (stage T1) [10]. The non-muscle invasive bladder cancer (NMIBC) shows significant patient-to-patient variability depending on disease characteristics: the probability of tumor recurrence at 1 year ranges from about $15 \%$ to $70 \%$ [11], and the probability of tumor progression at 5 years ranges from about $7 \%$ to $40 \%$ [12]. Predicting such behavior is clinically important as invasion bears a significant risk of metastasis and impaired survival $[13,14]$. Therefore, prediction of tumor recurrence and progression is critical for determining appropriate therapy and follow-up stratification. EMT is a key process in cancer development and progression [15-17]. So we aim to evaluate the status of Snail expression and their association with clinical outcomes of tumor recurrence and progression 
Table 1. Association of Snail Expression (Negative vs. Positive) in NMIBC Specimens With Clinical and Pathological Characteristics of 332 Patients Treated With TUR

\begin{tabular}{|c|c|c|c|c|}
\hline \multirow{2}{*}{ Variable } & \multirow{2}{*}{$\mathbf{N}$} & \multicolumn{2}{|c|}{ Snail expression status } & \multirow{2}{*}{$\mathbf{P}$} \\
\hline & & Negative & Positive & \\
\hline Total & 332 & $228(68.7 \%)$ & $104(31.3 \%)$ & - \\
\hline \multicolumn{5}{|l|}{ Age (years) } \\
\hline$\leq 65$ & $171(51.5 \%)$ & $119(75.3 \%)$ & $39(24.7 \%)$ & 0.013 \\
\hline$>65$ & $161(48.5 \%)$ & $109(62.6 \%)$ & $65(37.4 \%)$ & \\
\hline \multicolumn{5}{|l|}{ Gender } \\
\hline Female & $59(17.8 \%)$ & $42(71.2 \%)$ & $17(28.8 \%)$ & 0.646 \\
\hline Male & $273(82.2 \%)$ & $186(68.1 \%)$ & $87(31.9 \%)$ & \\
\hline \multicolumn{5}{|l|}{ Tumor size } \\
\hline$<3 \mathrm{~cm}$ & $221(66.6 \%)$ & $159(71.9 \%)$ & $62(28.1 \%)$ & 0.070 \\
\hline$\geq 3 \mathrm{~cm}$ & $111(33.4 \%)$ & $69(62.2 \%)$ & $42(37.8 \%)$ & \\
\hline \multicolumn{5}{|c|}{ Number of tumors } \\
\hline Single & $205(61.7 \%)$ & $154(75.1 \%)$ & $51(24.9 \%)$ & 0.001 \\
\hline Multiple & $127(38.3 \%)$ & $74(58.3 \%)$ & $53(41.7 \%)$ & \\
\hline \multicolumn{5}{|l|}{ Tumor stage } \\
\hline $\mathrm{Ta}$ & $204(61.4 \%)$ & $164(79.2 \%)$ & $43(20.8 \%)$ & $<0.0001$ \\
\hline $\mathrm{T} 1$ & $128(38.6 \%)$ & $64(51.2 \%)$ & $61(48.8 \%)$ & \\
\hline \multicolumn{5}{|l|}{ Tumor grade } \\
\hline G1 & $114(34.3 \%)$ & $99(86.8 \%)$ & $15(13.2 \%)$ & $<0.0001$ \\
\hline $\mathrm{G} 2$ & $168(50.6 \%)$ & $110(65.7 \%)$ & $58(43.3 \%)$ & \\
\hline G3 & $50(15.1 \%)$ & $18(36.7 \%)$ & $31(63.3 \%)$ & \\
\hline \multicolumn{5}{|c|}{ Concomitant CIS } \\
\hline No & $309(93.1 \%)$ & $217(70.7 \%)$ & $90(29.3 \%)$ & 0.006 \\
\hline Yes & $23(6.9 \%)$ & $11(44.0 \%)$ & $14(56.0 \%)$ & \\
\hline \multicolumn{5}{|l|}{ Recurrence } \\
\hline No & $213(64.2 \%)$ & $165(77.5 \%)$ & $48(22.5 \%)$ & $<0.0001$ \\
\hline Yes & $119(35.8 \%)$ & $63(52.9 \%)$ & $56(47.1 \%)$ & \\
\hline \multicolumn{5}{|l|}{ Progression } \\
\hline No & $292(88.0 \%)$ & $211(72.3 \%)$ & $81(27.7 \%)$ & $<0.0001$ \\
\hline Yes & $40(12.0 \%)$ & $17(42.5 \%)$ & $23(57.5 \%)$ & \\
\hline
\end{tabular}




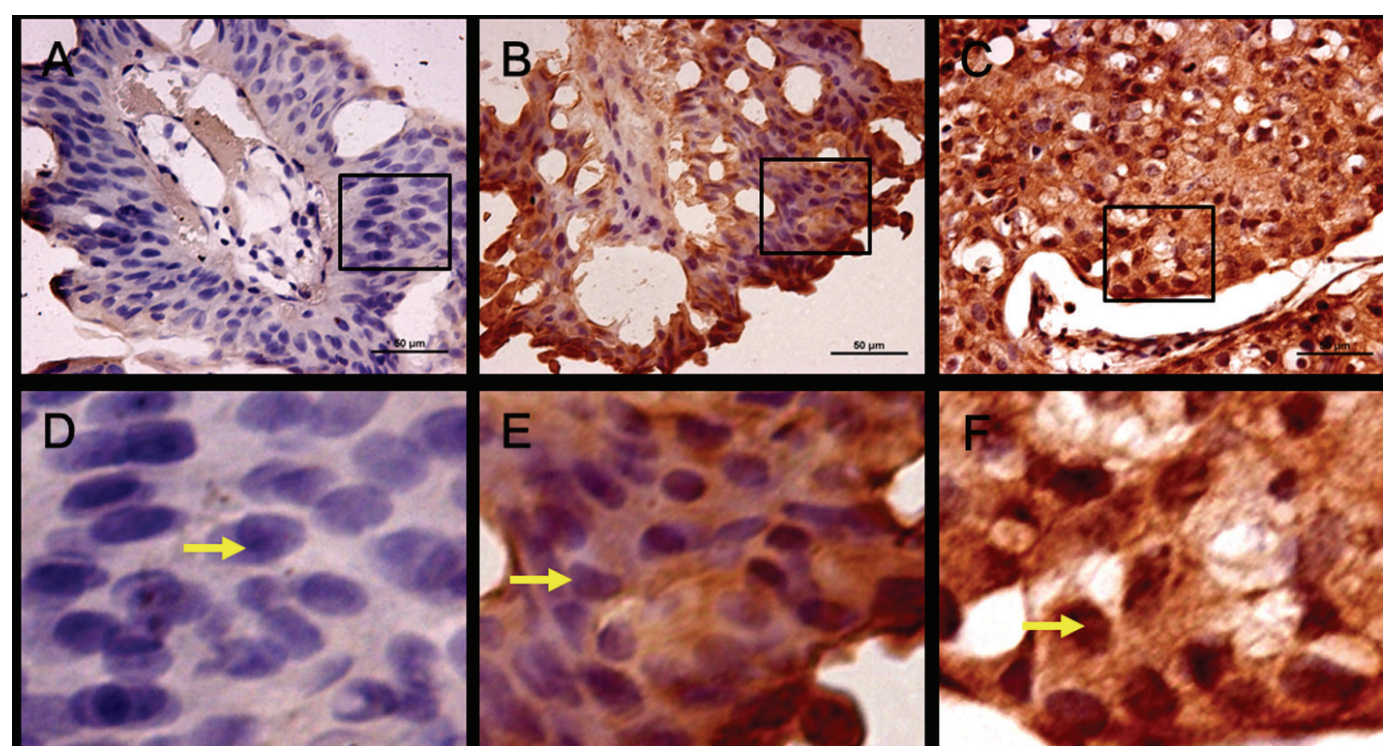

Figure 1. Expression and location of Snail in human bladder cancer. (A) Negative immunostaining for Snail, low grade. (B) Positive Snail immunostaining in cytoplasm, low grade. (C) Positive Snail immunostaining in cytoplasm and nucleus, high grade. (D-F) are part of (A-C) in the rectangle respectively ( $\times 400$, scale bar: $50 \mu \mathrm{m})$.

in a large cohort of patients with NMIBC.

\section{Materials and Methods}

The study cohort consisted of 332 patients treated with trans- urethral resection (TUR) of the bladder tumor and histopathologically confirmed to be NMIBC in our institution from 2004 to 2013. For each patient, comprehensive clinical data (Table 1) were collected from database of Huashan Hospital affiliated to Fudan University. Paraffin sections of tumors obtained from 332 cases were staged and graded according

Table 2. Univariate Cox Regression Model of Clinical and Pathological Variables Predicting Recurrence and Progression in 332 Patients in Our Cohort

\begin{tabular}{|c|c|c|c|c|}
\hline \multirow{2}{*}{ Variable } & \multicolumn{2}{|c|}{ Recurrence } & \multicolumn{2}{|c|}{ Progression } \\
\hline & HR & P value & HR & P value \\
\hline Age: $\leq 65$ years, $>65$ years & 1.160 & 0.420 & 0.792 & 0.469 \\
\hline Gender: male, female & 1.102 & 0.682 & 1.327 & 0.456 \\
\hline Number of tumors: single, multiple & 2.609 & $<0.0001$ & 1.840 & 0.055 \\
\hline Tumor size: $<3 \mathrm{~cm}, \geq 3 \mathrm{~cm}$ & 2.760 & $<0.0001$ & 4.657 & 0.0001 \\
\hline $\mathrm{T}$ category: Ta, $\mathrm{T} 1$ & 3.079 & $<0.0001$ & 7.953 & $<0.0001$ \\
\hline Grade: G1, G2 vs. G3 & 3.566 & $<0.0001$ & 8.624 & $<0.0001$ \\
\hline Carcinoma in situ: no, yes & 2.391 & 0.002 & 5.989 & $<0.0001$ \\
\hline Snail: negative, positive & 2.976 & $<0.0001$ & 4.713 & $<0.0001$ \\
\hline
\end{tabular}


Table 3. Multivariable Cox Regression Model of Clinical and Pathological Variables Predicting Recurrence and Progression in 332 Patients in Our Cohort

\begin{tabular}{lllll}
\hline & \multicolumn{2}{c}{ Recurrence } & \multicolumn{2}{c}{ Progression } \\
Variable & HR & P value & HR & P value \\
\cline { 2 - 5 } & & & & \\
\hline & & & & \\
\hline & & & & \\
Number of tumors: single, multiple & $1.91(1.31,2.79)$ & 0.001 & $2.33(0.69,2.58)$ & 0.394 \\
Tumor size: $<3$ cm, $\geq 3$ cm & $2.11(1.45,3.06)$ & $<0.0001$ & $2.92(1.48,5.76)$ & 0.002 \\
T category: Ta, T1 & $1.64(1.09,2.48)$ & 0.018 & $2.61(1.18,5.73)$ & 0.017 \\
Grade: G1, G2 vs. G3 & $1.63(1.03,2.58)$ & 0.037 & $2.36(1.11,5.05)$ & 0.026 \\
Carcinoma in situ: no, yes & $1.63(0.91,2.91)$ & 0.098 & $2.51(1.08,5.84)$ & 0.033 \\
Snail: negative, positive & $1.95(1.33,2.88)$ & 0.001 & $2.34(1.19,4.62)$ & 0.014 \\
\hline
\end{tabular}

to the 2002 TNM classification [18] and the 1973 WHO classification [19] by a pathologist with 10 years' experience in urology who was blinded to the clinical data for all patients.

The start time of the study was defined as the time after complete TUR. In all patients, cystoscopies were performed every 3 months for 2 years, then every 6 months to 5 years and annually thereafter, using a rigid endoscope with $70^{\circ}$ optics. The median follow-up duration was 43 months (range, 2 - 124 months). We define recurrence as the reappearance of disease (any grade, T category or CIS) after completion of TUR and progression as a tumor recurrence with either stage pT2 or higher disease in the bladder invasion.

For immunohistochemistry (IHC) analysis, $5 \mu \mathrm{m} \mathrm{sec-}$ tions from formalin-fixed paraffin-embedded tissue were taken on polylysine coated slides. Immunostaining was performed using anti-Snail (1:100, Abgent). Two independent pathologists assessed Snail immunostaining on coded slides (W.H. and C.Z.Q.). Representative areas were identified from the $H \& E$ sections and corresponding areas were scored using a conventional light microscope. Grades according to the intensity of the staining included: $0,1+, 2+$, and $3+$, while the percentages of positive cells were separated into: 0 $(0 \%), 1+(1-33 \%), 2+(34-66 \%)$, and $3+(67-100 \%)$. Scores of 0 and $1+$ were considered negative while $2+$ and $3+$ were considered positive.

\section{Results}

\section{Patients and IHC}

The characteristics of the 332 patients with NMIBC are summarized in Table 1. There were 273 male and 59 female pa- tients. The median age at diagnosis is 67 years (range 21 - 92 years), and median follow-up was 43 months (2 - 124 months). On follow-up, 119 (35.8\%) cases showed recurrence and $40(12.0 \%)$ cases had experienced a progression. The 1- and 5-year recurrence-free survival probabilities were $83.7 \%$ and $60.9 \%$, respectively. The 1 - and 5 -year progression-free survival probabilities were $95.4 \%$ and $83.4 \%$, respectively. In all, Snail positivity was present in 104 patients (31.3\%; Table 1 and Fig. 1B, E) while 228 patients presented negative (68.7\%; Table 1 and Fig. 1A, D).

\section{Association between Snail and clinicopathologic features}

We investigated the relationship between Snail and tumor stage.

Table 1 showed that Snail positivity was significantly associated with higher tumor stage and tumor grade $(\mathrm{P}<$ 0.0001 , respectively). Meanwhile age $>65$ years old, the presence of multifocality, and concomitant CIS were correlated with positive Snail staining too (each $\mathrm{P}<0.05$, Table 1). We found Snail staining was prone to weak in low risk (single, small size, and Ta/G1) tumors, and was cytosolic distribution. Interestingly, in higher tumor grade Snail staining was strong, but nucleus localization (Fig. 1B, C, E and F).

\section{Snail was proved to be an independent factor in predict- ing tumor recurrence and progression}

In univariate analysis, multifocality, tumor size, tumor stage, tumor grade and Snail staining correlated with recurrence, whereas tumor size, tumor stage, tumor grade, concomitant CIS and Snail staining correlated with muscle invasion 

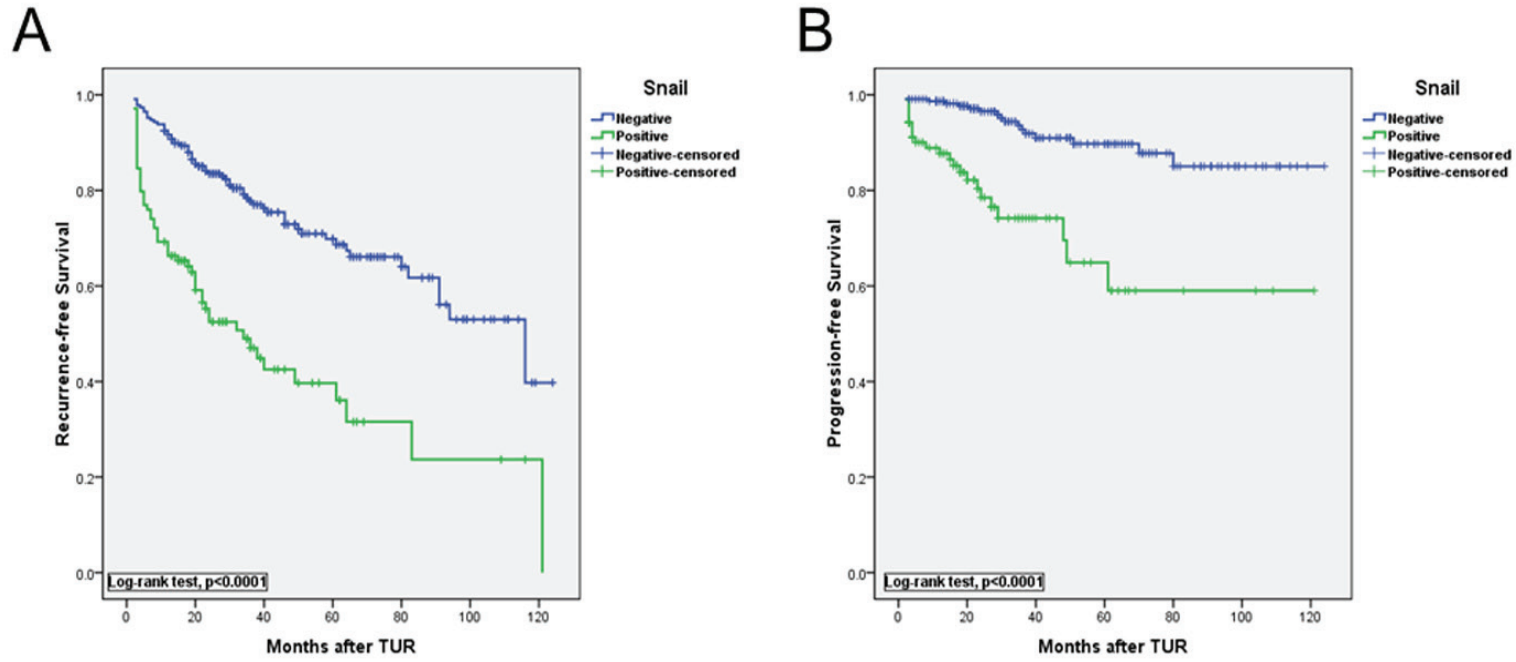

Figure 2. Kaplan-Meier curves show that recurrence-free $(A)$ and progression-free $(B)$ survival rates were significantly higher in high vs. low Snail group (log-rank test $\mathrm{P}<0.010$ and $\mathrm{P}<0.001$, respectively).

(Table 2). In multivariable analysis, Snail expression was an independent risk factor for predicting tumor recurrence (hazard ratio (HR): 1.95, $\mathrm{P}=0.001)$ and progression (HR: 2.34, $\mathrm{P}=0.014$; Table 3). Kaplan-Meier curves in Fig. 2 showed significant differences between negative and positive Snail expression for predicting tumor recurrence and progression $(\mathrm{P}<0.0001$, respectively, log-rank test $)$.

\section{Discussion}

Recently, histologic evidence of Snail expression in human tissue samples has also been reported in gastric cancer [20], hepatocellular carcinoma [21], and ovarian cancer [7] and is expected to serve as a novel prognostic indicator. Snail is a well-known $\mathrm{Zn}$-finger transcription factor that promotes EMT by repressing E-cadherin expression. It is known that Snail is phosphorylated by CK1 and subsequently GSK3b and degraded by $b$-TrCP-mediated ubiquitination $[2,3]$. In this study, we analyzed the expression profiles and subcellular localization of Snail and their relationship to the clinicopathological features of the NMIBC. We found that elevated levels of Snail expression and nucleus distribution were significantly associated with bladder tumor recurrence, which is suggestive that this marker may be an indicator of poor prognosis in NMIBC. Although faintish Snail expression emerges in low grade NMIBC, the Snail that located in cytoplasm is inactive and will be degraded by $b-\operatorname{TrCP}$ mediated ubiquitination. Inactive Snail has no contribution to tumor progression. Snail in nucleus of advanced tumor is active form to repress E-cadherin expression and promote EMT. The finding that Snail expression is associated with tumor recurrence conforms to the hypothesis that transcrip- tional regulators of EMT are important for advanced tumor features such as invasion and metastasis.

\section{Conclusions}

Results in our cohort show that positive expression of Snail is an independent predictor to determine primary NMIBC recurrence and progression.

\section{References}

1. Nieto MA. The snail superfamily of zinc-finger transcription factors. Nat Rev Mol Cell Biol. 2002;3(3):155166.

2. Zhou BP, Deng J, Xia W, Xu J, Li YM, Gunduz M, Hung MC. Dual regulation of Snail by GSK-3beta-mediated phosphorylation in control of epithelial-mesenchymal transition. Nat Cell Biol. 2004;6(10):931-940.

3. Xu Y, Lee SH, Kim HS, Kim NH, Piao S, Park SH, Jung YS, et al. Role of CK1 in GSK3beta-mediated phosphorylation and degradation of snail. Oncogene. 2010;29(21):3124-3133.

4. Dominguez D, Montserrat-Sentis B, Virgos-Soler A, Guaita S, Grueso J, Porta M, Puig I, et al. Phosphorylation regulates the subcellular location and activity of the snail transcriptional repressor. Mol Cell Biol. 2003;23(14):5078-5089.

5. Rosivatz E, Becker I, Specht K, Fricke E, Luber B, Busch $\mathrm{R}$, Hofler H, et al. Differential expression of the epithelial-mesenchymal transition regulators snail, SIP1, and twist in gastric cancer. Am J Pathol. 2002;161(5):18811891. 
6. Castro Alves C, Rosivatz E, Schott C, Hollweck R, Becker I, Sarbia M, Carneiro F, et al. Slug is overexpressed in gastric carcinomas and may act synergistically with SIP1 and Snail in the down-regulation of Ecadherin. J Pathol. 2007;211(5):507-515.

7. Blechschmidt K, Sassen S, Schmalfeldt B, Schuster T, Hofler H, Becker KF. The E-cadherin repressor Snail is associated with lower overall survival of ovarian cancer patients. Br J Cancer. 2008;98(2):489-495.

8. Jemal A, Bray F, Center MM, Ferlay J, Ward E, Forman D. Global cancer statistics. CA Cancer J Clin. 2011;61(2):69-90.

9. Parkin DM. The global burden of urinary bladder cancer. Scand J Urol Nephrol Suppl. 2008;(218):12-20.

10. Babjuk M, Oosterlinck W, Sylvester R, Kaasinen E, Bohle A, Palou-Redorta J, Roupret M. [EAU guidelines on non-muscle-invasive urothelial carcinoma of the bladder, the 2011 update]. Actas Urol Esp. 2012;36(7):389402.

11. Kondas J. [Factors affecting the recurrence rate and progression of superficial (Ta-T1) bladder tumors]. Orv Hetil. 1985;126(10):577-580.

12. Allard P, Bernard P, Fradet Y, Tetu B. The early clinical course of primary Ta and T1 bladder cancer: a proposed prognostic index. Br J Urol. 1998;81(5):692-698.

13. Soloway MS. It is time to abandon the "superficial" in bladder cancer. Eur Urol. 2007;52(6):1564-1565.

14. Bryan RT, Wallace DM. 'Superficial' bladder cancer - time to uncouple pT1 tumours from pTa tumours. BJU Int. 2002;90(9):846-852.

15. Bagnato A, Rosano L. Epithelial-mesenchymal transition in ovarian cancer progression: a crucial role for the endothelin axis. Cells Tissues Organs. 2007;185(13):85-94.

16. Moustakas A, Heldin CH. Signaling networks guiding epithelial-mesenchymal transitions during embryogenesis and cancer progression. Cancer Sci. 2007;98(10):1512-1520.

17. Hugo H, Ackland ML, Blick T, Lawrence MG, Clements JA, Williams ED, Thompson EW. Epithelial--mesenchymal and mesenchymal--epithelial transitions in carcinoma progression. J Cell Physiol. 2007;213(2):374-383.

18. FL G, et al. AJCC cancer staging Manual. 6th ed. New York: Springer Verlag. 2002.

19. FK M, S LH, T H. Histological typing of urinary bladder tumours. Geneva: World Health Organization: International Histological Classification of Tumors. 1973.

20. Rosivatz E, Becker KF, Kremmer E, Schott C, Blechschmidt K, Hofler H, Sarbia M. Expression and nuclear localization of Snail, an E-cadherin repressor, in adenocarcinomas of the upper gastrointestinal tract. Virchows Arch. 2006;448(3):277-287.

21. Jiao W, Miyazaki K, Kitajima Y. Inverse correlation between E-cadherin and Snail expression in hepatocellular carcinoma cell lines in vitro and in vivo. $\mathrm{Br} \mathrm{J}$ Cancer. 2002;86(1):98-101. 